\title{
Fisher Scientific
}

National Cancer Institute

\section{Source}

National Cancer Institute. Fisher Scientific. NCI Thesaurus. Code C126385.

A commercial provider of laboratory equipment, chemicals, supplies, and services used in scientific research, safety, healthcare, and education. 